\title{
Acute exercise increases triglyceride synthesis in skeletal muscle and prevents fatty acid-induced insulin resistance
}

\author{
Simon Schenk and Jeffrey F. Horowitz \\ Substrate Metabolism Laboratory, Division of Kinesiology, University of Michigan, Ann Arbor, Michigan, USA.
}

\begin{abstract}
Fatty acid oversupply is a key mediator of skeletal muscle insulin resistance in obesity, primarily via accumulation of fatty acid metabolites and activation of proinflammatory pathways. Herein, we demonstrate that fatty acid-induced insulin resistance in humans is completely prevented the day after 1 session of endurance exercise. Because skeletal muscle is the primary site for systemic glucose disposal and is highly susceptible to impaired insulin action by elevated fatty acid availability, we obtained skeletal muscle samples to investigate possible mechanisms mediating this protective effect of exercise. Prevention of fatty acid-induced insulin resistance after exercise accompanied enhanced skeletal muscle protein expression of key lipogenic enzymes and an increase in muscle triglyceride synthesis. Partitioning more fatty acids toward triglyceride synthesis within muscle reduced the accumulation of fatty acid metabolites and suppressed the proinflammatory response in skeletal muscle, as evidenced by decreased phosphorylation and

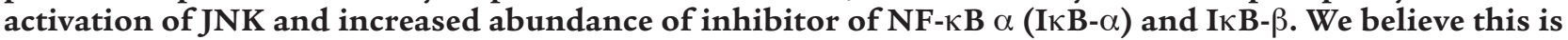
the first study to demonstrate that 1 session of exercise completely reverses fatty acid-induced insulin resistance in humans. Reversal of insulin resistance accompanied enhanced lipogenic capacity within skeletal muscle, reduced accumulation of highly bioactive fatty acid metabolites, and suppressed activation of proinflammatory pathways known to impair insulin action.
\end{abstract}

\begin{abstract}
Introduction
Excessive fatty acid mobilization found in abdominal obesity is a key mediator of many obesity-related metabolic complications, including insulin resistance (1-3). A high rate of fatty acid availability and subsequent uptake by skeletal muscle can augment intramuscular triglyceride (IMTG) accumulation $(1,3)$, and there is a strong correlation between IMTG concentration and the severity of insulin resistance in obese subjects and individuals with type 2 diabetes $(4,5)$. Recent studies, however, clarify that IMTG accumulation likely does not directly affect insulin action (6-9) but instead appears to be a biologically inert reservoir for fatty acids that can serve as a marker for high rates of fatty acid flux into muscle. Alternatively, it is now apparent that highly bioactive fatty acid metabolites, such as diacylglycerol and ceramide (and not IMTG), play a pivotal role in mediating fatty acid-induced insulin resistance in muscle (10-12). Considering the negative effects of diacylglycerol and ceramide on insulin action, we propose what we believe to be a novel hypothesis that, under conditions of high fatty acid flux, enhancing the partitioning of fatty acids entering the muscle toward IMTG synthesis will reduce the formation and accumulation of more damaging fatty acid metabolites within the cell, thereby improving insulin action (8).
\end{abstract}

\footnotetext{
Nonstandard abbreviations used: AMPK, AMP-activated protein kinase; $\mathrm{CHO}$, carbohydrate; COX-I, NADH-ubiquinol oxidoreductase; DGAT1, diacylglycerol

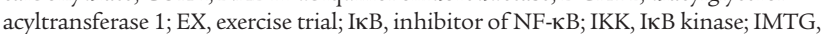
intramuscular triglyceride; IVGTT, i.v. glucose tolerance test; mGPAT, mitochondrial glycerol-3-phosphate acyltransferase; p-, phosphorylated; PGC-1, PPAR $\gamma$ coactivator $1 ; \mathrm{PRO}$, protein; RER, respiratory exchange ratio; SCD1, sterol CoA desaturase 1 ; SED sedentary trial; $\mathrm{S}_{\mathrm{i}}$, insulin sensitivity index; TZD, thiazolidinediones; $\mathrm{VCO}_{2}$, carbon dioxide production; $\mathrm{VO}_{2}$, oxygen uptake; $\mathrm{VO}_{2}$ peak, peak $\mathrm{VO}_{2}$

Conflict of interest: The authors have declared that no conflict of interest exists.
} Citation for this article: J. Clin. Invest. 117:1690-1698 (2007). doi:10.1172/JCI30566
IMTG synthesis is regulated by enzymes catalyzing a series of reactions that add activated fatty acid moieties (i.e., fatty acylCoA) to the glycerol-3-phosphate backbone. Two key proteins involved in this process are mitochondrial glycerol-3-phosphate (mGPAT) and diacylglycerol acyltransferase (DGAT), which catalyze the first and final steps in triglyceride synthesis, respectively. Overexpression of either mGPAT $(13)$ or $\operatorname{DGAT}(14,15)$ significantly increases triglyceride synthesis in numerous cell types. In fact, skeletal muscle-specific overexpression of the DGAT1 isoform results in a very large increase in IMTG concentration, which implies an important role for DGAT1 in regulating IMTG synthesis (14). Interestingly, DGAT1 overexpression in lung fibroblasts significantly decreased the accumulation of diacylglycerol, in parallel with increased triglyceride synthesis (15). However, while overexpression of DGAT1 and/or mGPAT clearly augment triglyceride synthesis, the interactive effects of increased mGPAT and DGAT expression, specifically in human skeletal muscle, on triglyceride synthesis and the accumulation of fatty acid metabolites and insulin sensitivity are not known.

Much of the inhibitory effect of intracellular fatty acid metabolites on insulin sensitivity works through activation of proinflammatory stress pathways within skeletal muscle. Increased activation

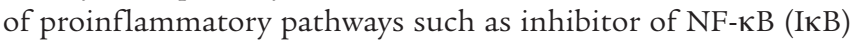

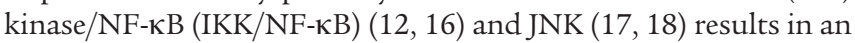
aberrant cascade of cellular events that ultimately leads to impaired insulin signaling and skeletal muscle insulin resistance. Indeed, activation of both the JNK and IKK/NF-KB pathways is elevated in skeletal muscle from obese and type 2 diabetic subjects and is strongly correlated with the severity of insulin resistance $(16,18-20)$. Therefore, reducing intracellular fatty acid metabolite accumulation by enhancing IMTG synthesis may improve insulin sensitivity via a reversal in the activation of these proinflammatory pathways. 


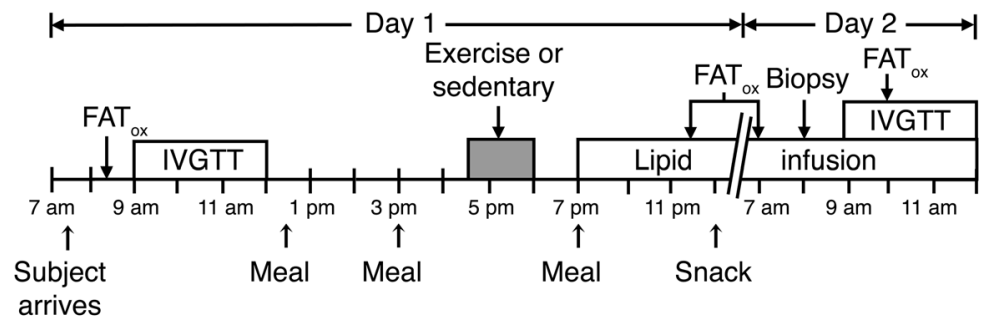

Figure 1

Timeline of events. FAT ox, measurement of whole-body fatty acid oxidation.

basal levels (Figure 2) in SED subjects, via an overnight infusion of lipid-plus-heparin, impaired insulin sensitivity index $\left(\mathrm{S}_{\mathrm{i}}\right)$ by approximately $30 \%$ the next day (Figure 3). However, EX subjects not only had a reverse in fatty acid-induced impairment in insulin sensitivity the next day, but also had a $25 \%$ increase in $\mathrm{S}_{\mathrm{i}}$ above basal levels (Figure 3). Metabolic data collected during the 90minute session of exercise is presented in Table 1 .

Acute exercise alters intramuscular fatty acid partitioning. Despite receiving exactly the same amount of lipid during the infusion in both trials, IMTG concentration was approximately $50 \%$ greater in EX compared with SED subjects $(59.8 \pm 3.9$ versus $39.8 \pm 3.4 \mathrm{mmol} / \mathrm{kg}$ dry

Key evidence leading to the consensus that IMTG may not be directly involved in impairing insulin sensitivity is the finding that individuals who exercise regularly are acutely insulin sensitive despite having high IMTG concentrations $(6,9)$, commonly referred to as the "athlete's paradox" (6). Importantly, only 1 session of exercise markedly improves insulin sensitivity $(21,22)$, and this improvement also occurs in obesity (23). One session of exercise also increases IMTG synthesis and storage, particularly when fat availability is high (7-9). Therefore, by preferentially repartitioning fatty acids that have entered the muscle cell toward storage as IMTG, a single session of exercise may reduce the partitioning of fatty acids toward more metabolically "unfavorable" routes (i.e., excessive formation and accumulation of fatty acid metabolites). Notably, this proposed mechanism may only be relevant under conditions when fatty acid availability is high, and thus this may be very important for the exercise-induced improvements in insulin sensitivity found in obesity. A possible mechanism for increased IMTG synthesis following exercise could be increased expression of the lipogenic enzymes DGAT1 and MGPAT, although whether acute exercise enhances the expression of these lipogenic proteins in human muscle is unknown.

In the present study, we tested the hypothesis that a single session of exercise in human subjects would protect against fatty acid-induced insulin resistance the next day. In addition, we examined whether a single session of exercise can enhance partitioning of excess fatty acids toward IMTG synthesis and away from the excessive formation of more metabolically bioactive metabolites that are known to activate proinflammatory pathways, and thereby inhibit insulin signaling. To test this hypothesis, all subjects participated in 2 separate trials (Figure 1). The only difference between the 2 trials was whether the subjects remained sedentary throughout the trial (SED) or performed 90 minutes of moderateintensity exercise in the afternoon of day 1 (EX). We administered an overnight lipid infusion in both trials and measured insulin sensitivity using an i.v. glucose tolerance test (IVGTT). Overall, our results indicate that EX subjects had an enhanced capacity for IMTG synthesis, reduced the accumulation of fatty acid metabolites in skeletal muscle, and suppressed downstream activation of proinflammatory pathways. This repartitioning of fatty acids within skeletal muscle may be an important mechanism responsible for the observed exercise-mediated protection against fatty acid-induced insulin resistance.

\section{Results}

One session of exercise prevents fatty acid-induced insulin resistance. As was found in previous reports $(1,3,24)$, we observed that increasing plasma fatty acid concentration approximately 4-fold above weight, $P<0.001$; Figure $4 \mathrm{~A})$. In conjunction with an enhanced partitioning of fatty acids toward IMTG and protection against fatty acid-induced insulin resistance, skeletal muscle diacylglycerol concentration was significantly reduced in EX compared with SED $(P<0.05)$, and there was a strong trend toward reduced ceramide concentration as well $(P=0.056)$ (Figure $4, \mathrm{~B}$ and $\mathrm{C}$ ). This is consistent with our hypothesis that a single session of exercise will increase the partitioning of fatty acids toward storage as IMTG, rather than toward metabolite accumulation. Also, muscle glycogen concentration was approximately $30 \%$ lower in EX compared with SED $(P<0.001$; Figure 4D).

Acute exercise enhances the capacity for triglyceride synthesis in skeletal muscle. To investigate putative mechanisms responsible for increased IMTG storage after exercise, we measured the protein abundance of mGPAT and DGAT1. In parallel with increased IMTG concentration after exercise, skeletal muscle abundance of mGPAT and DGAT1 was significantly higher in EX compared with SED, by approximately $35 \%(P<0.02)$ and $25 \%(P<0.01)$, respectively (Figure 5, A and $\mathrm{B}$ ). In addition, the protein expression of sterol CoA desaturase 1 (SCD1), which desaturates fatty acids and enhances their incorporation into triglycerides (25-27), was approximately $35 \%$ higher in EX compared with SED ( $P<0.01$; Figure 5C).

Skeletal muscle proinflammatory stress is lower after exercise. Considering the tight link between fatty acid availability, skeletal muscle inflammation, and insulin resistance $(12,16-20)$, we measured the

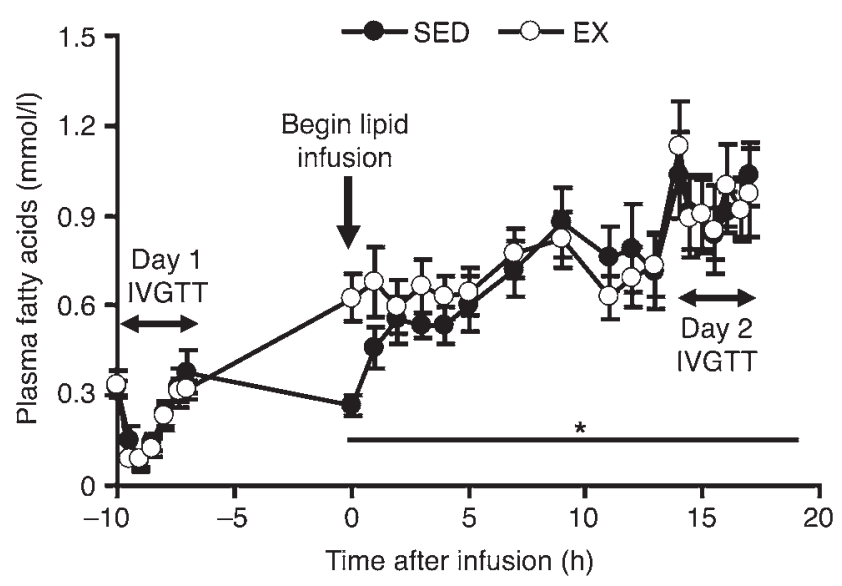

Figure 2

Plasma fatty acid concentrations before and during a lipid-plus-heparin infusion. Values are mean $\pm \operatorname{SEM}(n=8)$. ${ }^{*} P<0.05$ versus overnight fasted value on day 1 for each group. There were no significant differences between SED and EX, except at 0 hours $(P<0.05)$. 


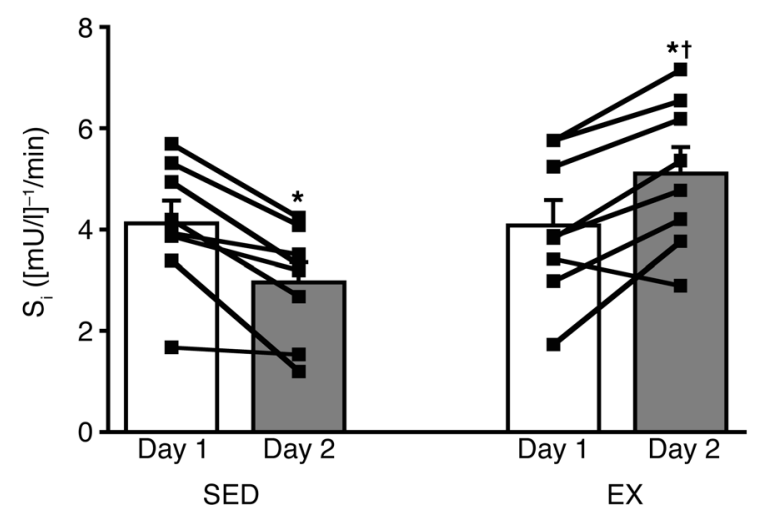

Figure 3

A single session of exercise prevents lipid-induced insulin resistance. $\mathrm{S}_{i}$ was measured before (day 1) and during (day 2) a lipid-plus-heparin infusion. Lines connected by dots indicate individual subjects' day 1 and day $2 S_{i}$ levels. Values are mean \pm SEM $(n=8)$. ${ }^{*} P<0.001$ versus day 1 within respective trial; ${ }^{\dagger} P<0.001$ versus day 2 SED.

activation of the proinflammatory JNK and IKK/NF- $\mathrm{KB}$ pathways. Although total JNK-1 abundance was not different between trials $(0.92 \pm 0.14$ for EX versus $0.83 \pm 0.08$ AU for SED; $P=N S)$, phosphorylation/activation of JNK (p-JNK) was approximately $50 \%$ lower in EX compared with SED $(P<0.05$; Figure 6A). Additionally, activation of the IKK/NF- $\mathrm{KB}$ pathway was significantly lower during EX compared with SED, as evidenced by higher abundance of IкB- $\alpha(P<0.001)$ and IкB- $\beta(P<0.05$; Figure 6 , B and $C)$, which are key inhibitors of the IKK/NF- $\kappa \mathrm{B}$ pathway. Therefore, changes in the activation of these important proinflammatory pathways may further explain how a single session of exercise protected against fatty acid-induced insulin resistance.

Exercise increases the ratio of catalytic subunits to regulatory subunits of $P I 3 K$. To gain further insight into possible mechanisms mediating improved insulin sensitivity in EX we measured the ratio of catalytic $(\mathrm{p} 110 \beta)$ to regulatory $(\mathrm{p} 85 \alpha / \beta)$ subunits of PI3K, which has been shown to be an excellent index of insulin sensitivity $(19,28,29)$. While there was no significant difference in $\mathrm{p} 110 \beta$ protein abundance between EX and SED ( $P=$ NS; Figure 7A), in parallel with the improved insulin sensitivity after EX, the protein abundance of $\mathrm{p} 85 \alpha / \beta$ was significantly lower in EX compared with $\operatorname{SED}(P<0.05$; Figure $7 \mathrm{~B})$. As a result, the ratio of the catalytic subunits to regulatory subunits was approximately $40 \%$ greater in EX compared with SED $(P<0.05$; Figure $7 \mathrm{C})$.

Exercise increases the partitioning of fatty acids toward oxidation. Along with increased partitioning of fatty acids toward IMTG, wholebody fatty acid oxidation was significantly higher in EX compared with SED (Figure 8A). This increase in fat oxidation was not due to an increase in energy expenditure for EX subjects because the rate of oxygen consumption (i.e., $\mathrm{VO}_{2}$ ) was not significantly different between trials (Figure 8B). Instead the increase in fat oxidation appeared to be due to an increased contribution of fat to energy production, as evidenced by a significant decrease in the respiratory exchange ratio (RER) $(P<0.05$; Figure $8 \mathrm{C})$. Despite these differences in fatty acid oxidation and RER, the protein abundance in skeletal muscle of PPAR $\gamma$ coactivator 1 (PGC-1; Figure 8D), a master regulator of mitochondrial biogenesis, and NADH-ubiquinol oxidoreductase (COX-I; data not shown), a marker of mitochondrial density, was not different between EX and SED.

\section{Discussion}

High rates of systemic fatty acid availability and uptake by skeletal muscle is a key mediator of insulin resistance in obesity $(1-3,12,24)$. Importantly, this fatty acid-induced insulin resistance is largely determined by the partitioning of excess fatty acids within the muscle cell toward storage, oxidation, or the accumulation of intracellular fatty acid metabolites (e.g., diacylglycerol, ceramide) $(12,17,26,27,30)$. Herein, we found that a single session of exercise completely reversed the fatty acid-induced impairment in insulin sensitivity. To our knowledge, this is the first study to demonstrate that lipid-induced insulin resistance in humans can be prevented by a physiological intervention, such as exercise, and corroborates results in highfat diet-fed rats $(31,32)$. Interestingly, we found that this reversal in fatty acid-induced insulin resistance was accompanied by an exercise-mediated enhancement of the lipogenic capacity of skeletal muscle, as evidenced by increased protein expression of mGPAT, DGAT1, and SCD1 and a resultant increase in the disposal of fatty acids toward IMTG synthesis. In parallel with this increase in triglyceride synthesis, there was a reduced accumulation of fatty acid metabolites within skeletal muscle. In turn, activation of the proinflammatory JNK and IKK/NF-kB pathways was suppressed during the lipid infusion in EX subjects compared with the same lipid infusion in SED subjects.

In agreement with our hypothesis that increasing the lipogenic capacity of skeletal muscle can improve insulin sensitivity, several studies have recently reported that enhancing triglyceride synthesis in rat skeletal muscle and in L6 myotubes attenuated the accumulation of diacylglycerol and/or ceramide and preserved insulin sensitivity in response to elevated lipid availability $(26,27,30)$. Similarly, in humans, Bachmann et al. (3) demonstrated that subjects with the greatest capacity to partition fatty acids toward IMTG synthesis had the smallest impairment in insulin sensitivity when fat availability was increased through either diet or a lipid-plus-heparin infusion. Interestingly, part of the insulin-sensitizing effect of thiazolidinediones (TZD), which are able to prevent fatty acid-induced insulin resistance (33), was recently attributed to an enhanced capacity for IMTG synthesis. In a recent study by Todd et al. (30), TZD treatment of rodents on a high-fat diet augmented muscle triglyceride synthesis, reduced the accumulation of ceramide, prevented activation of inflammatory pathways, and ameliorated high-fat diet-induced insulin resistance. Therefore, our findings suggest that acute exercise and TZD treatment may prevent fatty acid-induced insulin resistance through a similar mechanism.

Although IMTG synthesis is accelerated after exercise (7-9), the molecular mechanisms controlling this adaptation are not completely understood. Herein, we provide exciting insight into the possible mechanisms responsible for enhanced IMTG synthesis after exercise. We demonstrate for what we believe to be the first

\section{Table 1}

Metabolic data during exercise

$\begin{array}{lccc} & \text { Treadmill } & \text { Bicycle ergometer } & \begin{array}{c}\text { Average } \\ \mathrm{VO}_{2}(\mathrm{ml} / \mathrm{min})\end{array} \\ 1420 \pm 101 & 1389 \pm 87 & 1404 \pm 82 \\ \% \mathrm{VO}_{2} \text { peak } & 66.5 \pm 2.8 & 65.4 \pm 2.1 & 65.9 \pm 0.9 \\ \mathrm{RER} & 0.91 \pm 0.01 & 0.92 \pm 0.02 & 0.91 \pm 0.01\end{array}$

Values are mean \pm SEM. 
A

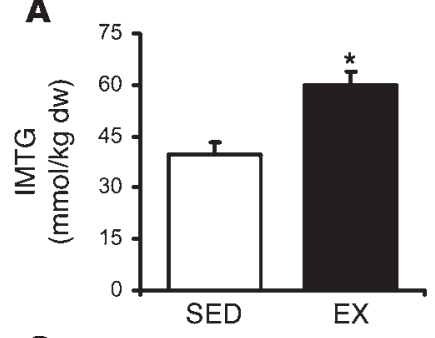

C

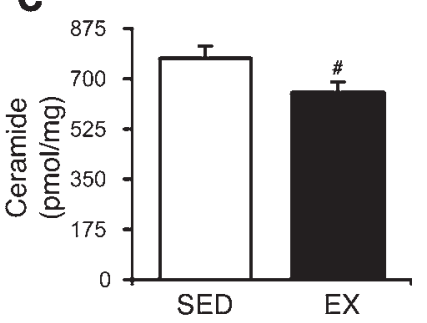

B

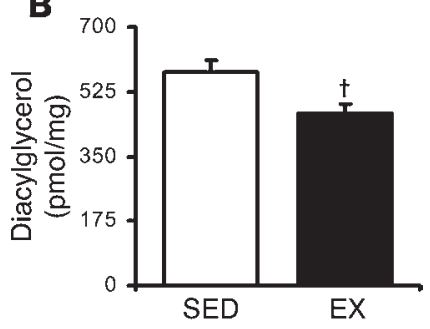

D

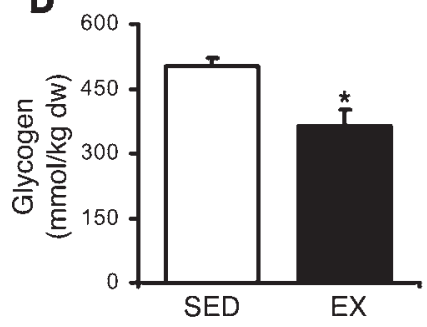

Figure 4

Exercise enhances the partitioning of fatty acids toward IMTG and reduces the accumulation of fatty acid metabolites in skeletal muscle. (A) IMTG, (B) diacylglycerol, (C) ceramide, and (D) muscle glycogen concentrations the morning after an overnight infusion of lipid-plus-heparin. Values are mean $\pm \operatorname{SEM}(n=8) .{ }^{*} P<0.001,{ }^{\dagger} P<0.05,{ }^{\#} P=0.056$ versus SED. dw, dry weight.

time that a single session of exercise increased the protein abundance of mGPAT, DGAT, and SCD1 in human skeletal muscle in parallel with increased IMTG synthesis. In rodents, exercise increases the gene and protein expression of SREBP-1c $(34,35)$, which is a key regulator of several lipogenic enzymes including mGPAT (36) and SCD1 (37). Importantly, overexpression of DGAT1 or SCD1 in vivo $(14)$ or in vitro $(26,27)$ results in large increases in triglyceride synthesis and also reduces diacylglycerol accumulation $(15,26,27)$, while overexpression of mGPAT in liver cells increases the incorporation of exogenous fatty acids into triglyceride (13). Overall, we provide compelling support for the hypothesis that a single session of exercise induces changes within skeletal muscle that promote the partitioning of excess fatty acids toward IMTG, resulting in a parallel reduction in the accumulation of fatty acid metabolites.

Accumulation of diacylglycerol and/or ceramide in skeletal muscle can activate proinflammatory pathways such as IKK/ NF-KB and JNK, and recent research has highlighted the importance of these proinflammatory responses in mediating skeletal muscle insulin resistance. Activation of IKK/NF-KB and JNK is elevated in obesity and type 2 diabetes (18-20), while inactivation of these pathways protects against lipid-induced insulin resistance $(16,18)$. In addition, a recent study in humans found that endurance exercise training reduced activation of the IKK/NF-KB pathway, as indicated by increased abundance of IкB- $\alpha$ and IкB- $\beta$, and significantly improved insulin action (20). Similarly, a recent study in rats found that a single session of exercise prevented high-fat diet-induced insulin resistance in parallel with reduced activation of JNK and increased IкB- $\alpha$ abundance (31). Therefore, our findings corroborate the notion that exercise can play a significant role in suppressing a lipid-induced proinflammatory response in skeletal muscle. Our findings, and those of Ropelle et al. (31), also suggest that much of the positive effect of exercise on proinflammatory stress in skeletal muscle is due to the most recent session of exercise, rather than an effect of chronic exercise training and a subsequent improvement in fitness.

In our study, the single session of exercise did not just prevent the reduction in insulin sensitivity when subjects were exposed to an approximate 4-fold elevation in fatty acid availability, but insulin sensitivity was increased $25 \%$ above basal levels. Along with our results showing the important contribution of alterations in fatty acid partitioning on insulin sensitivity, a reduction in muscle glycogen concentration after exercise is known to be a key mediator of enhanced insulin sensitivity after exercise $(38,39)$. Accordingly, it is likely that the approximately $30 \%$ lower muscle glycogen concentration in EX compared with SED on day 2 played an important role in enhancing insulin sensitivity. The decreased abundance of the regulatory subunit of PI3K and increased PI3K catalytic subunit to regulatory subunit ratio in EX compared with SED may also contribute to improved insulin sensitivity by enhancing insulin signaling $(19,28,29)$. It is also interesting to note that the PI3K p85 $\alpha / \beta$ subunit has been found to directly regulate JNK activity, such that a decrease in p85 abundance leads to a decrease in JNK activation (29). Therefore, in addition to the important effects that reduced accumulation of fatty acid metabolites has on the proinflammatory response in
A

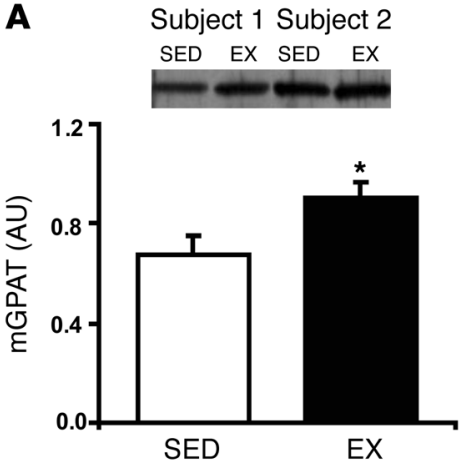

B

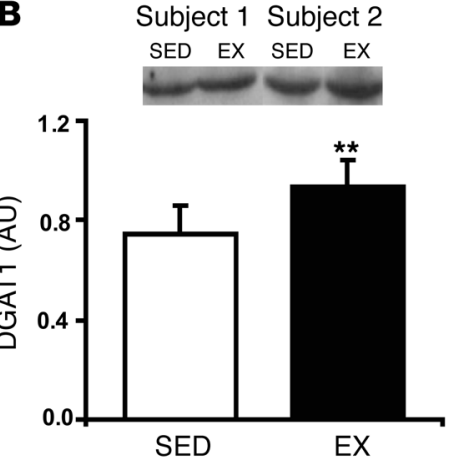

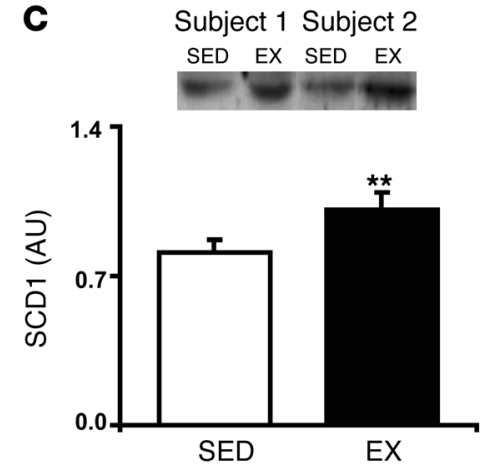

Figure 5

Exercise increases the lipogenic capacity of muscle. Protein abundance of (A) mGPAT, (B) DGAT1, and (C) SCD1 the morning after an overnight infusion of lipid-plus-heparin. Insets are representative western blots for 2 subjects. Values are mean \pm SEM $(n=8)$. ${ }^{\star} P<0.02$, ${ }^{* *} P<0.01$ versus SED. 
A

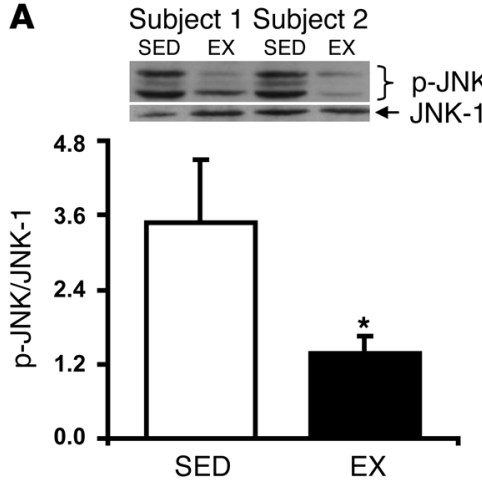

B

Subject 1 Subject 2 SED EX SED EX

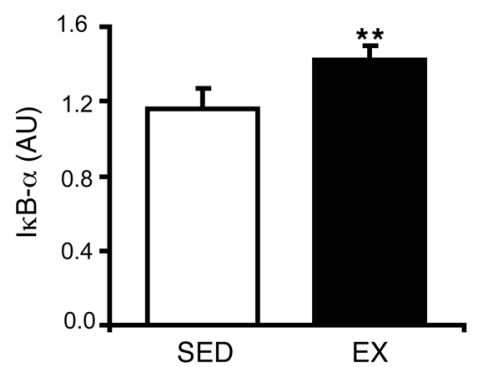

C

Subject 1 Subject 2 SED EX SED EX

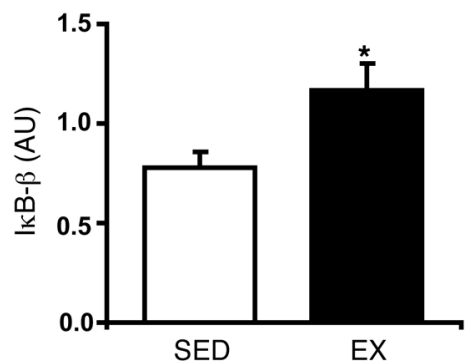

Figure 6

Skeletal muscle proinflammatory response during a lipid infusion is lower after exercise. (A) p-JNK, corrected for JNK-1 protein abundance, and protein abundance of $\mathrm{I} \mathrm{KB}-\alpha(\mathbf{B})$ and $\mathrm{I} \mathrm{\kappa} B-\beta(\mathbf{C})$ the morning after an overnight infusion of lipid-plus-heparin. Insets are representative western blots for 2 subjects. Values are mean \pm SEM $(n=8) .{ }^{*} P<0.05,{ }^{*} P<0.001$ versus SED.

EX compared with SED, a decreased abundance of $\mathrm{p} 85 \alpha / \beta$ may also lead to decreased JNK phosphorylation/activation.

In addition to repartitioning fatty acids toward storage in muscle, EX also showed increased whole-body fatty acid oxidation for at least 16 hours after exercise. Previous studies also demonstrate that fatty acid oxidation is enhanced after an acute bout of exercise (40), especially when dietary fat availability is increased (41, 42 ). We hypothesize that the reduction in muscle glycogen concentration after exercise was the major mediator of enhanced fatty acid oxidation in EX, as has been previously reported $(41,42)$. In addition, acute exercise decreases the activity of pyruvate dehydrogenase for at least 18 hours after exercise (43), which should also favor enhanced fatty acid oxidation. An exercise-mediated increase in AMP-activated protein kinase (AMPK) activity can also augment fat oxidation $(44,45)$. However, because AMPK activity returns to pre-exercise levels within 1-3 hours following acute exercise (44, 45 ) it is unlikely that enhanced AMPK function was responsible for changes in substrate metabolism the day after exercise. Our finding that PGC-1 protein abundance, which is increased by AMPK activation (46), was similar in EX and SED supports the notion that downstream effects of AMPK were not enhanced the day after the exercise session.
Recent reports have suggested that low fatty acid oxidative capacity may be a key determinant for the development of insulin resistance $(47,48)$. Theoretically, an increase in fatty acid oxidative capacity might improve the "mismatch" between fatty acid availability and associated metabolism, thereby reducing the accumulation of fatty acid metabolites. However, the absolute increase in fat oxidation in our study $(\sim 70 \mu \mathrm{mol}$ fatty acid/min $)$ did not approach the estimated increase in fatty acid availability from the lipid infusion ( $400 \mu \mathrm{mol}$ fatty acid/ $\mathrm{min})$, which is comparable to the rate of fatty acid availability found in abdominal obesity (49). Therefore, while it is likely that increasing fatty acid partitioning toward oxidation after acute exercise may contribute to the exercise-mediated protection against fatty acid-induced insulin resistance, this contribution is relatively small.

It is important to acknowledge that our measurement of insulin sensitivity (i.e., IVGTT) measures whole-body insulin sensitivity, and therefore, we were unable to assess the exact contribution of skeletal muscle to the enhancement in insulin sensitivity after exercise. However, it has been found that approximately $85 \%$ of measured and calculated $S_{i}$ from the IVGTT is a result of insulin increasing glucose uptake into skeletal muscle (50). Additionally, fatty acid-induced insulin resistance has been found to be primari-
A
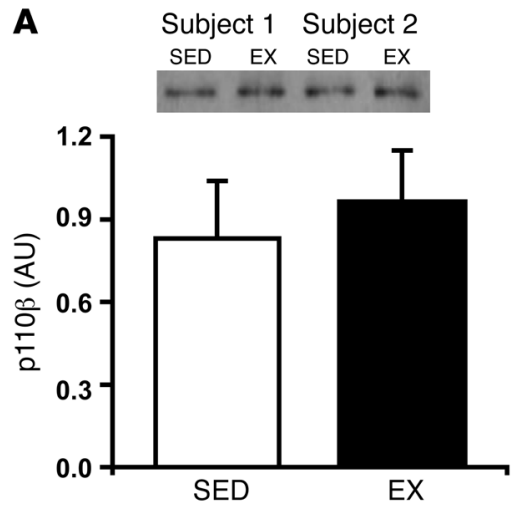

B

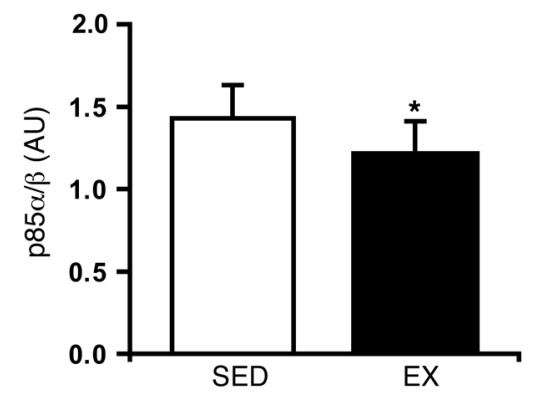

C

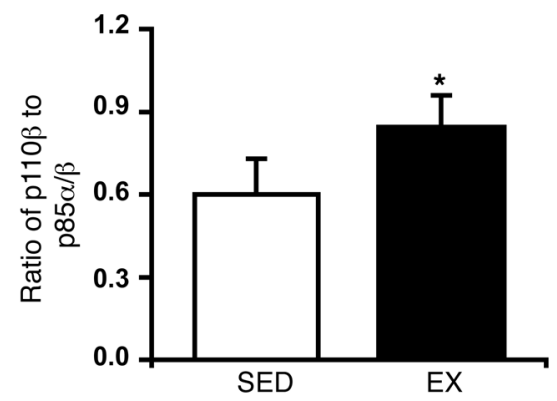

Figure 7

The ratio of PI3K catalytic subunits to regulatory subunits is higher after exercise. Protein abundance of the PI3K catalytic subunits ( $1110 \beta)(\mathbf{A})$ and regulatory subunits $(\mathrm{p} 85 \alpha / \beta)(B)$ and $($ C) the ratio of PI3K catalytic subunits to regulatory subunits the morning after an overnight infusion of lipid-plus-heparin. Insets are representative western blots for 2 subjects. Values are mean \pm SEM $(n=8)$. ${ }^{*} P<0.05$ versus SED. 
A
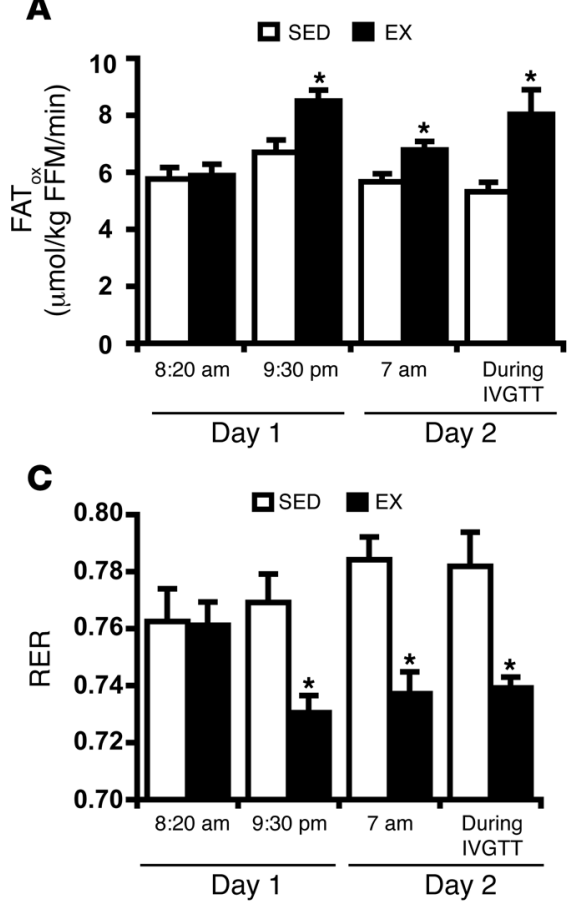

B

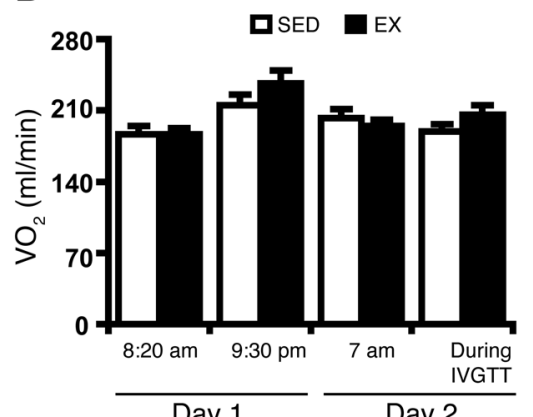

Day 1
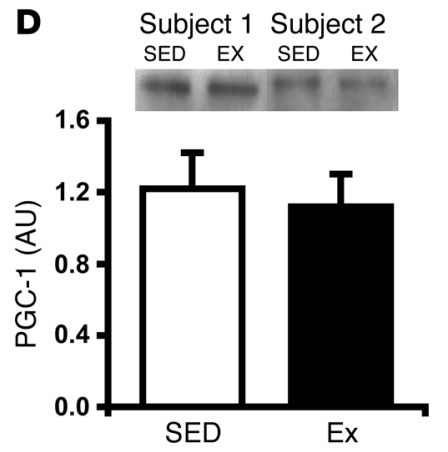

Figure 8

Partitioning of fatty acids toward oxidation during a lipid-plus-heparin infusion is enhanced after exercise. (A) Whole-body fatty acid oxidation, (B) resting $\mathrm{VO}_{2},(\mathbf{C}) \mathrm{RER}$, and (D) skeletal muscle protein abundance of PGC-1 before and during a lipid-plus-heparin infusion. Inset figures are representative Western blots for 2 subjects. FFM, fat-free mass. Values are mean $\pm \operatorname{SEM}(n=8) .{ }^{*} P<0.05$ versus SED.

ly due to defects in skeletal muscle insulin action $(12,24)$, and exercise exerts its metabolic effects specifically in the muscles recruited during exercise $(21,39)$. Also, the findings we did observe in skeletal muscle after exercise (i.e., a reduction in fatty acid metabolites and reduced activation of proinflammatory pathways) have been clearly linked with improvements in skeletal muscle insulin signaling $(16-18,30)$. Therefore, we propose that skeletal muscle is likely responsible for much of the improvement in fatty acid-induced insulin resistance that we observed in EX. We do acknowledge that changes in hepatic insulin sensitivity after acute exercise may contribute to the change in $\mathrm{S}_{\mathrm{i}}$, but this contribution would be relatively small (51). Moreover, considering the strong relationship between fatty acid availability, obesity, and metabolic health, our finding that a single session of exercise completely reversed whole-body fatty acid-induced insulin resistance in humans has profound clinical implications.

In summary, we have demonstrated that a single session of exercise can protect against fatty acid-induced insulin resistance. Our novel findings indicate that this protective effect of exercise was accompanied by an increased lipogenic capacity of muscle and a resultant increase in partitioning of excess fatty acids toward triglyceride synthesis in muscle. This repartitioning of fatty acids after exercise toward IMTG synthesis and oxidation reduced the accumulation of bioactive fatty acid metabolites, which are known to increase the activation of proinflammatory pathways in skeletal muscle and induce insulin resistance. Because high fatty acid availability found in obesity is associated with elevated proinflammatory stress $(16,18-20)$, the data from

this study outline an important putative mechanism by which regularly performed endurance exercise may help to improve insulin resistance in individuals with excessive endogenous fatty acid availability such as that seen in obesity.

\section{Methods}

Subjects. Eight non-obese women participated in this study. All subjects were premenopausal and were considered to be in good health after a comprehensive medical examination, which included a history and physical examination, a 12-lead electrocardiogram, standard blood and urine tests, an aerobic fitness test (i.e., peak $\mathrm{VO}_{2}\left[\mathrm{VO}_{2}\right.$ peak $]$ ), and assessment of body composition (i.e., dual-energy $\mathrm{x}$-ray absorptiometry). No subject was taking regular medications (except birth control). Subject characteristics are presented in Table 2 . All subjects were fully informed of the possible risks associated with the study and signed an informed consent document, which was approved by the University of Michigan Institutional Review Board.

Preliminary testing. Whole-body fat mass (FM) and fat-free mass (FFM) were determined using dualenergy $\mathrm{x}$-ray absorptiometry (Lunar DPX DEXA Scanner; Lunar Radiation Corp). $\mathrm{VO}_{2}$ peak was measured during cycle ergometer exercise (Lode Excaliber; Physio-Dyne Instrument Corp.). The protocol consisted of a 4-minute warm-up, after which the work rate was progressively increased every minute until volitional fatigue.

Experimental protocol. All subjects participated in 2 separate experimental trials. The trials differed only by whether the subjects performed 1 bout of exercise or remained sedentary. Trials were randomized, separated by at least 7 days, and were performed during the follicular phase of the subjects' menstrual cycle. A timeline of events is presented in Figure 1 . The evening before each trial, subjects ingested a standard-

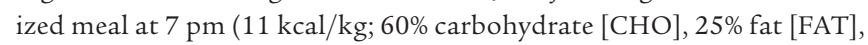
$15 \%$ protein [PRO]) and a standardized high-CHO snack at $10 \mathrm{pm}$ ( $3 \mathrm{kcal} / \mathrm{kg}$; 95\% CHO, 2\% FAT, 3\% PRO). All meals were the same in both trials. After an overnight fast, subjects arrived at the University of Michigan Medical Center at 7 am for admission to the General Clinical Research Center. After admission, subjects rested in their hospital bed. At 7:45 am, an intravenous catheter was placed in an antecubital vein of one arm for glucose and insulin administration during the IVGTT and another was placed in a contralateral hand vein. At 8:20

\section{Table 2}

\section{Subject characteristics}

\section{Characteristic}

Age, yr

Weight, kg

Height, cm

Body mass index, $\mathrm{kg} / \mathrm{m}^{2}$

Body fat, \%

$\mathrm{VO}_{2}$ peak, $\mathrm{ml} / \mathrm{kg} / \mathrm{min}$

\section{Value}

$26 \pm 2$

$58.8 \pm 2.3$

$164 \pm 2$

$22 \pm 1$

$29.4 \pm 3.0$

$36.4 \pm 2.1$
Values are mean \pm SEM. 
am, resting $\mathrm{VO}_{2}$ and carbon dioxide production $\left(\mathrm{VCO}_{2}\right)$ were measured (DeltaTrac II, Sensormedics; VIASYS Healthcare Inc.) for 20 to $30 \mathrm{~min}$ utes to calculate resting whole-body fatty acid oxidation. At 9 am, subjects underwent an IVGTT in order to assess insulin sensitivity using the minimal model method (52). Briefly, at 9 am, subjects were i.v. injected with a bolus of glucose ( $300 \mathrm{mg} / \mathrm{kg}$ body weight), and at 9:20 am, subjects received an i.v. bolus of insulin $(0.02 \mathrm{IU} / \mathrm{kg})$. Blood samples were taken at frequent time points for 3 hours after the glucose bolus, as previously described (8). Afterward, the IVGTT subjects ate standardized meals at 12:15 pm and $3 \mathrm{pm}$ (both meals were $7 \mathrm{kcal} / \mathrm{kg}$; $60 \% \mathrm{CHO}$, $25 \% \mathrm{FAT}, 15 \% \mathrm{PRO})$. Beginning at $4: 30 \mathrm{pm}$, subjects either remained sedentary or performed 90 minutes of exercise at approximately $65 \%$ $\mathrm{VO}_{2}$ peak. The exercise consisted of 45 minutes of treadmill exercise followed immediately by 45 minutes of exercise on a cycle ergometer. $\mathrm{VO}_{2}$ and $\mathrm{VCO}_{2}$ were measured using indirect calorimetry (Physio-Dyne Instrument Corp.) during minutes 3-8 and 30-35 of each mode of exercise, and appropriate adjustments were made to the exercise intensity as necessary. Subjects showered between $6 \mathrm{pm}$ and $7 \mathrm{pm}$. In both trials, a constant-rate infusion $(0.55 \mathrm{ml} / \mathrm{kg} / \mathrm{h})$ of a lipid-plus-heparin solution (lipid, Liposyn II 20\%; Abbott Laboratories; heparin, $5 \mathrm{U} / \mathrm{kg} / \mathrm{h}$; Elkins-Sinn Inc.) was started at $7 \mathrm{pm}$ and was continued until the end of the study the next day (day 2). Overnight blood samples were taken every 1-2 hours during the lipid infusion for measurement of plasma fatty acid concentration. At $7 \mathrm{pm}$ and $10 \mathrm{pm}$, a standardized meal and high-CHO snack were served, respectively. These meals were identical to the meals provided the night before. For the remainder of the study, subjects remained fasted and were only allowed to drink water. Wholebody fatty acid oxidation was measured at 9:30 pm on day 1 and 7 am on day 2 for 20 to 30 minutes. At 8 am on day 3, a muscle biopsy was obtained from the vastus lateralis, using the percutaneous biopsy technique. Muscle samples were dissected free of adipose and connective tissue, rinsed in saline, blotted dry, and then frozen in liquid nitrogen. After the muscle biopsy, a third catheter was placed in an antecubital vein for administration of glucose and insulin for the second IVGTT, which was started at $9 \mathrm{am}$, as described above. Sixty minutes after the glucose injection, whole-body fatty acid oxidation was again measured to assess insulin-stimulated fatty acid oxidation.

Blood sampling and plasma metabolite and insulin concentrations. All blood samples were collected in tubes containing EDTA $(25 \mathrm{mg} / \mathrm{ml})$. Samples were then centrifuged at $1,572 \mathrm{~g}$ for 20 minutes, and the plasma was removed and stored at $-80^{\circ} \mathrm{C}$ for later analysis. Plasma glucose (glucose oxidase assay; Thermo Scientific) and fatty acid (NEFA C assay kit; Wako Chemicals USA) concentrations were measured by colorimetric assay. Plasma insulin concentration was measured by radioimmunoassay (Human Insulin Specific RIA; Millipore).

$S_{i}$ calculation. The $S_{i}$ was calculated from least-squares fitting of the insulin and glucose concentration curves from the IVGTT using the Minimal Model Millennium (version 6.02; MinMod Inc.) computer analysis software. It must be noted that $S_{i}$ does not allow us to differentiate insulin sensitivity among different tissues. However, $S_{\mathrm{i}}$ has been found to closely match insulin-stimulated glucose disposal into skeletal muscle as measured by the hyperinsulinemic-euglycemic clamp technique $(50,53)$. Therefore changes in $\mathrm{S}_{\mathrm{i}}$ are likely reflective of changes in skeletal muscle insulin sensitivity. Furthermore, because muscle contraction induces a profound improvement in skeletal muscle insulin sensitivity $(21,22)$, specifically in the muscle activated during exercise $(21,39)$, we attribute most of the change in $S_{i}$ after exercise to improvements in insulin sensitivity within skeletal muscle. We do acknowledge that changes in hepatic insulin sensitivity after acute exercise may contribute to the change in $\mathrm{S}_{\mathrm{i}}$, but this contribution is relatively small (51).
Muscle metabolite analysis. For IMTG analysis, triglycerides were extracted from freeze-dried muscle in 2:1 chloroform:methanol and dried under $\mathrm{N}_{2}$ gas. The triglyceride was then saponified in $4 \%$ ethanol-KOH solution at $75^{\circ} \mathrm{C}$, and the glycerol concentration was measured fluorometrically (8). Muscle glycogen was determined by measuring the liberation of glucose from freeze-dried muscle after acid hydrolysis in $\mathrm{HCl}$ (8). Diacylglycerol and ceramide were analyzed radioactively using diacylglycerol kinase and $\left[\gamma^{-32} \mathrm{P}\right]$ ATP. Briefly, lipids were extracted from muscle using chloroform $/ \mathrm{methanol} / \mathrm{HCl}$. Butylated hydroxytoluene $(0.01 \%)$ was included in the extraction solution as an antioxidant, and $\left[{ }^{3} \mathrm{H}\right]$ triolein $(0.25 \mu \mathrm{Ci})$ was used as an internal control for triglyceride recovery. The lipid phase was isolated, dried under $\mathrm{N}_{2}$ stream, and then redissolved in $7.5 \%$ octyl- $\beta$-Dglucoside containing $5 \mathrm{mM}$ cardiolipin and $1 \mathrm{mM}$ diethylenetriamine pentaacetate. The diacylglycerol kinase reaction was performed at room temperature in $50 \mathrm{mM}$ imidazole. $\mathrm{HCl}, 12.5 \mathrm{mM} \mathrm{NaCl}, 12.5 \mathrm{mM} \mathrm{MgCl}$, $1 \mathrm{mM}$ EGTA (pH 6.6), $2 \mu \mathrm{g}$ diacylglycerol kinase (Sigma-Aldrich), $1 \mathrm{mM}$ ATP, $2 \mathrm{mCi}\left[\gamma_{-}{ }^{32} \mathrm{P}\right] \mathrm{ATP}$, and $40 \mu \mathrm{g}$ sample extract (Sigma-Aldrich). The reaction was stopped after 30 minutes by the addition of chloroform/ methanol (v/v, 2:1) and $1 \% \mathrm{HClO}_{4}$. Lipids were extracted and washed twice with $1 \% \mathrm{HClO}_{4}$ and then resolved by thin layer chromatography (TLC) (Partisil K6 adsorption TLC plates, catalog no. LK6D; Whatman; mobile phase containing chloroform/methanol/acetic acid, $\mathrm{v} / \mathrm{v}, 65: 15: 5$ ). Bands corresponding to phosphatidic acid and ceramide-1-phosphate were scraped into a scintillation vial for radioactivity measurement. $\left[{ }^{3} \mathrm{H}\right]$ triolein bands from the same TLC plates were quantified the same way and were used as controls for lipid recovery.

Muscle protein analysis. Frozen muscle samples were homogenized in ice-cold $0^{\circ} \mathrm{C}$ lysis buffer (50 mM HEPES, pH 7.5, $150 \mathrm{mM} \mathrm{NaCl}, 10 \mathrm{mM}$ sodium pyrophosphate, $10 \mathrm{mM} \mathrm{NaF}, 2 \mathrm{mM}$ EDTA, 1\% Nonidet P-40, 10\% glycerol), which contained appropriate protease inhibitors (Pefabloc SC and Complete; Roche Applied Science; phosphatase inhibitor cocktail, P-5726; Sigma-Aldrich). Homogenates were solubilized for 1 hour at $4{ }^{\circ} \mathrm{C}$ by end-over-end rotation and were then centrifuged at $16,000 \mathrm{~g}$ for $20 \mathrm{~min}$ utes to remove insoluble material. The protein concentration of the supernatant was determined by the bicinchoninic acid assay (Pierce Biotechnology), and samples were then aliquoted and stored at $-80^{\circ} \mathrm{C}$. For western blotting analysis, samples $(30 \mu \mathrm{g})$ were separated by SDS-PAGE and were then transferred to nitrocellulose $(0.45 \mu \mathrm{m})$ for 2 hours at $200 \mathrm{~mA}$ in an ice bath. After blocking, membranes were probed for mGPAT (a kind gift from R.A. Coleman, University of North Carolina Chapel Hill, Chapel Hill, North Carolina, USA), DGAT1 (a kind gift from R.V. Farese Jr., The J. David Gladstone Institutes, San Francisco, California, USA), SCD1 (catalog no. SCD11-A; Alpha Diagnostic International), p-JNK (catalog no. 9251; Cell Signaling Technology), JNK-1 (catalog no. sc-474; Santa Cruz Biotechnology Inc.), IкB- $\alpha$ (catalog no. 9242; Cell Signaling Technology), ІкB- $\beta$ (catalog no. sc-945; Santa Cruz Biotechnology Inc.), p $85 \alpha / \beta$ (catalog no. 06-195; Millipore), p110 $\beta$ (catalog no. sc-602; Santa Cruz Biotechnology Inc.), PGC-1 (catalog no. 516557; EMD Biosciences), and COX-I (catalog no. A21344; Invitrogen). Membranes were then incubated in the appropriate secondary antibody for 60 minutes and were developed with enhanced chemiluminescence (Amersham Biosciences). Bands were quantified by densitometry (Fluor Chem SP imaging system; Alpha Innotech Corp.). Band densities on each blot were expressed relative to a standard (muscle from lean female), which was run in duplicate on all blots.

Substrate oxidation. Fatty acid oxidation was calculated from $\mathrm{VO}_{2}$ and $\mathrm{VCO}_{2}$ measurements using the equations of Frayn (54).

Statistics. A repeated measures 2-way ANOVA with Tukey's procedure was used to test for significant differences in $\mathrm{S}_{\mathrm{i}}$, fatty acid oxidation, and the overnight plasma fatty acid concentration. A repeated measures 1-way ANOVA with Tukey's procedure was used to test for significant differences 
in muscle metabolites and muscle protein abundance/phosphorylation. Statistical analyses were performed using SigmaStat for Windows (version 3.0.1a; Systat Software Inc.). Statistical significance was defined as $P<0.05$. All results are presented as mean \pm SEM.

\section{Acknowledgments}

This study was supported by the American Diabetes Association (grant 1-03-JF-10) and the NIH General Clinical Research Centers (grant M01-RR00042). We are very grateful to Cara R. Shrivastava and Sacha L. Hamady for their outstanding work with subject scheduling and recruiting; Naila Goldenberg, Matthew P. Harber, and Nicolas D. Knuth for assistance during data collection; Yi-Hao Yu and Li Liu for analysis of skeletal muscle diacylglycerol and ceramide concentrations; the nursing and dietary staff of the
General Clinical Research Center at the University of Michigan University Hospital for their excellent assistance; and the Chemistry Core of the Michigan Diabetes Research and Training Center (grant 5P60 DK20572 from NIDDK, NIH) for measurement of the plasma insulin concentrations. Finally, we thank the subjects for their enthusiastic participation.

Received for publication October 6, 2006, and accepted in revised form February 19, 2007.

Address correspondence to: Jeffrey F. Horowitz, Substrate Metabolism Laboratory, Division of Kinesiology, University of Michigan, 401 Washtenaw Ave., Ann Arbor, Michigan 48109-2214, USA. Phone: (734) 647-1076; Fax: (734) 936-1925; E-mail: jeffhoro@umich.edu.
1. Boden, G., Lebed, B., Schatz, M., Homko, C., and Lemieux, S. 2001. Effects of acute changes of plasma free fatty acids on intramyocellular fat content and insulin resistance in healthy subjects. Diabetes. 50:1612-1617.

2. Santomauro, A.T., et al. 1999. Overnight lowering of free fatty acids with Acipimox improves insulin resistance and glucose tolerance in obese diabetic and nondiabetic subjects. Diabetes. 48:1836-1841.

3. Bachmann, O.P., et al. 2001. Effects of intravenous and dietary lipid challenge on intramyocellular lipid content and the relation with insulin sensitivity in humans. Diabetes. 50:2579-2584.

4. Goodpaster, B.H., Thaete, F.L., Simoneau, J.A., and Kelley, D.E. 1997. Subcutaneous abdominal fat and thigh muscle composition predict insulin sensitivity independently of visceral fat. Diabetes. 46:1579-1585.

5. Krssak, M., et al. 1999. Intramyocellular lipid concentrations are correlated with insulin sensitivity in humans: a $1 \mathrm{H}$ NMR spectroscopy study. Diabetologia. 42:113-116.

6. Goodpaster, B.H., He, J., Watkins, S., and Kelley, D.E. 2001. Skeletal muscle lipid content and insulin resistance: evidence for a paradox in endurance-trained athletes. J. Clin. Endocrinol. Metab. 86:5755-5761.

7. Fox, A.K., Kaufman, A.E., and Horowitz, J.F. 2004. Adding fat calories to meals after exercise does not alter glucose tolerance. J. Appl. Physiol. 97:11-16.

8. Schenk, S., Cook, J.N., Kaufman, A.E., and Horowitz, J.F. 2005. Postexercise insulin sensitivity is not impaired after an overnight lipid infusion. Am. J. Physiol. Endocrinol. Metab. 288:E519-E525.

9. Decombaz, J., et al. 2001. Postexercise fat intake repletes intramyocellular lipids but no faster in trained than in sedentary subjects. Am. J. Physiol. Regul. Integr. Comp. Physiol. 281:R760-R769.

10. Chavez, J.A., and Summers, S.A. 2003. Characterizing the effects of saturated fatty acids on insulin signaling and ceramide and diacylglycerol accumulation in 3T3-L1 adipocytes and C2C12 myotubes. Arch. Biochem. Biophys. 419:101-109.

11. Yu, C., et al. 2002. Mechanism by which fatty acids inhibit insulin activation of insulin receptor substrate-1 (IRS-1)-associated phosphatidylinositol 3-kinase activity in muscle. J. Biol. Chem. 277:50230-50236.

12. Itani, S.I., Ruderman, N.B., Schmieder, F., and Boden, G. 2002. Lipid-induced insulin resistance in human muscle is associated with changes in diacylglycerol, protein kinase C, and IkappaB-alpha. Diabetes. 51:2005-2011.

13. Igal, R.A., Wang, S., Gonzalez-Baro, M., and Coleman, R.A. 2001. Mitochondrial glycerol phosphate acyltransferase directs the incorporation of exogenous fatty acids into triacylglycerol. J. Biol. Chem. 276:42205-42212.

14. Roorda, B.D., et al. 2005. DGAT1 overexpres- sion in muscle by in vivo DNA electroporation increases intramyocellular lipid content. J. Lipid Res. 46:230-236.

15. Bagnato, C., and Igal, R.A. 2003. Overexpression of diacylglycerol acyltransferase-1 reduces phospholipid synthesis, proliferation, and invasiveness in simian virus 40-transformed human lung fibroblasts. J. Biol. Chem. 278:52203-52211.

16. Yuan, M., et al. 2001. Reversal of obesity- and dietinduced insulin resistance with salicylates or targeted disruption of Ikkbeta. Science. 293:1673-1677.

17. Watt, M.J., Hevener, A., Lancaster, G.I., and Febbraio, M.A. 2006. Ciliary neurotrophic factor prevents acute lipid-induced insulin resistance by attenuating ceramide accumulation and phosphorylation of JNK in peripheral tissues. Endocrinology. 147:2077-2085.

18. Hirosumi, J., et al. 2002. A central role for JNK in obesity and insulin resistance. Nature. 420:333-336.

19. Bandyopadhyay, G.K., Yu,J.G., Ofrecio,J., and Olefsky, J.M. 2005. Increased p85/55/50 expression and decreased phosphotidylinositol 3-kinase activity in insulin-resistant human skeletal muscle. Diabetes. 54:2351-2359.

20. Sriwijitkamol, A., et al. 2006. Reduced skeletal muscle inhibitor of kappaBbeta content is associated with insulin resistance in subjects with type 2 diabetes: reversal by exercise training. Diabetes. 55:760-767.

21. Richter, E.A., Mikines, K.J., Galbo, H., and Kiens, B. 1989. Effect of exercise on insulin action in human skeletal muscle. J. Appl. Physiol. 66:876-885.

22. Perseghin, G., et al. 1996. Increased glucose transport-phosphorylation and muscle glycogen synthesis after exercise training in insulin-resistant subjects. N. Engl. J. Med. 335:1357-1362.

23. Devlin, J.T., and Horton, E.S. 1985. Effects of prior high-intensity exercise on glucose metabolism in normal and insulin-resistant men. Diabetes. 34:973-979.

24. Dresner, A., et al. 1999. Effects of free fatty acids on glucose transport and IRS-1-associated phosphatidylinositol 3-kinase activity. J. Clin. Invest. 103:253-259.

25. Listenberger, L.L., Ory, D.S., and Schaffer, J.E. 2001. Palmitate-induced apoptosis can occur through a ceramide-independent pathway. J. Biol. Chem. 276:14890-14895.

26. Lee, J.S., et al. 2006. Saturated, but not n- 6 polyunsaturated, fatty acids induce insulin resistance: role of intramuscular accumulation of lipid metabolites. J. Appl. Physiol. 100:1467-1474.

27. Pinnamaneni, S.K., Southgate, R.J., Febbraio, M.A., and Watt, M.J. 2006. Stearoyl CoA desaturase 1 is elevated in obesity but protects against fatty acidinduced skeletal muscle insulin resistance in vitro. Diabetologia. 49:3027-3037.

28. Mauvais-Jarvis, F., et al. 2002. Reduced expression of the murine p $85 \alpha$ subunit of phosphoinositide 3- kinase improves insulin signaling and ameliorates diabetes. J. Clin. Invest. 109:141-149. doi:10.1172/ JCI200213305.

29. Ueki, K., et al. 2003. Positive and negative roles of p85 alpha and p85 beta regulatory subunits of phosphoinositide 3-kinase in insulin signaling. J. Biol. Chem. 278:48453-48466.

30. Todd, M.K., Watt, M.J., Le, J., Hevener, A.L., and Turcotte, L.P. 2007. Thiazolidinediones enhance skeletal muscle triacylglycerol synthesis while protecting against fatty acid-induced inflammation and insulin resistance. Am. J. Physiol. Endocrinol. Metab. 292:E485-E493.

31. Ropelle, E.R., et al. 2006. Reversal of diet-induced insulin resistance with a single bout of exercise in the rat: the role of PTP1B and IRS-1 serine phosphorylation. J. Physiol. 577:997-1007.

32. Oakes, N.D., et al. 1997. Diet-induced muscle insulin resistance in rats is ameliorated by acute dietary lipid withdrawal or a single bout of exercise: parallel relationship between insulin stimulation of glucose uptake and suppression of long-chain fatty acyl-CoA. Diabetes. 46:2022-2028.

33. Hevener, A.L., Reichart, D., Janez, A., and Olefsky, J. 2001. Thiazolidinedione treatment prevents free fatty acid-induced insulin resistance in male wistar rats. Diabetes. 50:2316-2322.

34. Ikeda, S., et al. 2002. Up-regulation of SREBP$1 \mathrm{c}$ and lipogenic genes in skeletal muscles after exercise training. Biochem. Biophys. Res. Commun. 296:395-400.

35. Nadeau, K.J., et al. 2006. Exercise training and calorie restriction increase SREBP-1 expression and intramuscular triglyceride in skeletal muscle. Am. J. Physiol. Endocrinol. Metab. 291:E90-E98.

36. Ericsson, J., Jackson, S.M., Kim, J.B., Spiegelman, B.M., and Edwards, P.A. 1997. Identification of glycerol-3-phosphate acyltransferase as an adipocyte determination and differentiation factor 1and sterol regulatory element-binding proteinresponsive gene. J. Biol. Chem. 272:7298-7305.

37. Shimomura, I., Shimano, H., Korn, B.S., Bashmakov, Y., and Horton, J.D. 1998. Nuclear sterol regulatory element-binding proteins activate genes responsible for the entire program of unsaturated fatty acid biosynthesis in transgenic mouse liver. J. Biol. Chem. 273:35299-35306.

38. Wojtaszewski, J.F., et al. 2003. Insulin signalling: effects of prior exercise. Acta Physiol. Scand. 178:321-328.

39. Cartee, G.D., et al. 1989. Prolonged increase in insulin-stimulated glucose transport in muscle after exercise. Am. J. Physiol. 256:E494-E499.

40. Votruba, S.B., Atkinson, R.L., Hirvonen, M.D., and Schoeller, D.A. 2002. Prior exercise increases subsequent utilization of dietary fat. Med. Sci. Sports Exerc. 34:1757-1765.

41. Schrauwen, P., Lichtenbelt, W.D., Saris, W.H., and Westerterp, K.R. 1998. Fat balance in obese 
subjects: role of glycogen stores. Am. J. Physiol. 274:E1027-E1033.

42. Schrauwen, P., van Marken Lichtenbelt, W.D., Saris, W.H., and Westerterp, K.R. 1997. Role of glycogen-lowering exercise in the change of fat oxidation in response to a high-fat diet. Am. J. Physiol. 273:E623-E629.

43. Kimber, N.E., Heigenhauser, G.J., Spriet, L.L., and Dyck, D.J. 2003. Skeletal muscle fat and carbohydrate metabolism during recovery from glycogen-depleting exercise in humans. J. Physiol. 548:919-927.

44. Wojtaszewski, J.F., et al. 2003. Regulation of 5'AMPactivated protein kinase activity and substrate utilization in exercising human skeletal muscle. Am. J. Physiol. Endocrinol. Metab. 284:E813-E822.

45. Wojtaszewski, J.F., Mourtzakis, M., Hillig, T., Saltin, B., and Pilegaard, H. 2002. Dissociation of AMPK activity and ACCbeta phosphorylation in human muscle during prolonged exercise. Biochem. Biophys. Res. Commun. 298:309-316.

46. Terada, S., et al. 2002. Effects of low-intensity prolonged exercise on PGC-1 mRNA expression in rat epitrochlearis muscle. Biochem. Biophys. Res. Commun. 296:350-354.

47. Petersen, K.F., Dufour, S., Befroy, D., Garcia, R., and Shulman, G.I. 2004. Impaired mitochondrial activity in the insulin-resistant offspring of patients with type 2 diabetes. N. Engl. J. Med. 350:664-671.

48. Mootha, V.K., et al. 2003. PGC-1alpha-responsive genes involved in oxidative phosphorylation are coordinately downregulated in human diabetes. Nat. Genet. 34:267-273.

49. Horowitz, J.F., et al. 1999. Effect of short-term fasting on lipid kinetics in lean and obese women. Am. J. Physiol. 276:E278-E284.

50. Bergman, R.N., Prager, R., Volund, A., and Olefsky, J.M. 1987. Equivalence of the insulin sensitivity index in man derived by the minimal model method and the euglycemic glucose clamp. J. Clin. Invest. 79:790-800.

51. Hamilton, K.S., et al. 1996. Effect of prior exercise on the partitioning of an intestinal glucose load between splanchnic bed and skeletal muscle. J. Clin. Invest. 98:125-135.

52. Yang, Y.J., Youn, J.H., and Bergman, R.N. 1987. Modified protocols improve insulin sensitivity estimation using the minimal model. Am. J. Physiol. 253:E595-E602.

53. Saad, M.F., et al. 1994. A comparison between the minimal model and the glucose clamp in the assessment of insulin sensitivity across the spectrum of glucose tolerance. Insulin Resistance Atherosclerosis Study. Diabetes. 43:1114-1121.

54. Frayn, K.N. 1983. Calculation of substrate oxidation rates in vivo from gaseous exchange. J. Appl. Physiol. 55:628-634. 